\title{
Clinicopathological characteristics of vascular endothelial growth factor expression in uveal melanoma: A meta-analysis
}

\author{
MENG YANG ${ }^{1}$, XIAOCONG KUANG ${ }^{2}$, YANBIN PAN $^{3}$, MEILE TAN $^{3}$, BINZHU LU $^{3}$, \\ JIAN $\mathrm{LU}^{3}$, QIUMEI CHENG ${ }^{1}$ and JIANMIN $\mathrm{LI}^{3}$ \\ ${ }^{1}$ Postgraduate School and ${ }^{2}$ Department of Pathophysiology, Guangxi Medical University, Nanning, Guangxi 530021; \\ ${ }^{3}$ Department of Dermatology, The Third Affiliated Hospital of Guangxi Medical University, \\ Nanning, Guangxi 530000, P.R. China
}

Received June 6, 2013; Accepted August 22, 2013

DOI: $10.3892 / \mathrm{mco} .2014 .247$

\begin{abstract}
Angiogenesis is a potential prognostic factor that has been extensively investigated in patients with uveal melanoma (UM). Vascular endothelial growth factor (VEGF) expression is crucial in angiogenesis. However, there have been conflicting data regarding the clinicopathological data in UM. A meta-analysis was performed of all the germane literature to assess the clinicopathological characteristics of VEGF expression by combining separately estimated odds ratio (OR) values. Our combined results demonstrated that, according to the available studies, the expression of VEGF in UM was significantly higher compared to normal tissue [338 patients and 99 controls; $O R=16.15$, 95\% confidence interval $(\mathrm{CI}): 8.65-30.12, \mathrm{P}<0.00001]$. When stratifying the studies by age (315 patients; $\mathrm{OR}=2.08$, 95\% CI: $1.19-3.62, \mathrm{P}=0.01$ ), cell type (423 patients; $\mathrm{OR}=0.54$, 95\% CI: $0.32-0.90, \mathrm{P}=0.02)$, tumor size (222 patients; $\mathrm{OR}=0.30$, 95\% CI: $0.14-0.68, \mathrm{P}=0.004)$ and scleral invasion (248 patients; $\mathrm{OR}=0.34$, 95\% CI: 0.15-0.78, $\mathrm{P}=0.01)$, significant clinicopathological information was provided. Our results indicated that VEGF expression in UM patients was significantly higher compared to that observed in controls. It was also significantly higher in patients who presented with scleral invasion and those who were aged $<50$ years. In addition, VEGF expression was higher in mixed-cell type and epithelioid-cell type UM and in patients with large-sized tumors.
\end{abstract}

\section{Introduction}

Uveal melanoma (UM) is a malignant tumor of the eye involving the iris, choroid or ciliary body (collectively referred

Correspondence to: Dr Jianmin Li, Department of Dermatology, The Third Affiliated Hospital of Guangxi Medical University, 13 Dan Cun Road, Nanning, Guangxi 530031, P.R. China E-mail: 4830380@163.com

Key words: uveal melanoma, vascular endothelial growth factor expression, clinicopathological characteristics, angiogenesis to as the uvea). It is the most common intraocular tumor, with an incidence of 5-6 cases per 1,000,000 individuals (1). There is a reported incidence of 4-6 cases per 1,000,000 individuals annually in the US and Europe (2). UM spreads through the blood and exhibits a high degree of malignancy.

Angiogenesis is a prognostic factor that has been extensively investigated in patients with UM (3). It involves the formation of new blood vessels from the endothelium of the existing vasculature. When a new tumor reaches a size of 1-2 $\mathrm{mm}$, its further growth requires the formation of new blood vessels, which may lead to the development of metastases (4). As one of the most important biological markers, vascular endothelial growth factor (VEGF) is an endothelial cell mitogen and permeability factor that is potently angiogenic in vivo (5). VEGF induces the proliferation, differentiation and migration of vascular endothelial cells. VEGF is also required for haematopoiesis in malignant tumors, which favors primary tumor growth and metastasis (6).

Targeting constitutive VEGF and/or its receptors has been an attractive approach for cancer therapy. However, the most widely investigated clinicopathological characteristics associated with VEGF refer to variables including tumor size, largest tumor diameter (LTD), depth of invasion, lymph node status and vascular invasion. Although implicated in the pathogenesis of UM, the results on the correlation between VEGF and these factors have been conflicting and inconclusive (7-10).

It has not been elucidated whether the differences in these studies were due to the limited size of the samples or genuine heterogeneity. Therefore, in order to gain a full insight into the clinicopathological characteristics of VEGF expression in patients with UM, we enrolled data from studies of medical centers. The clinicopathological significance of our present analysis may enable a better understanding of the natural history of UM. In addition, the use of VEGF may be converted from candidate to the routine clinical setting as a predictor of the outcome of individual patients.

\section{Materials and methods}

Literature search. A search was conducted through PubMed, Embase, Cochrane Library, CNKI, VIP and Wanfang electronic databases, without language restrictions. The search was 
based on the terms 'melanoma', 'vascular endothelial growth factor' or 'VEGF', 'melanoma' and 'neovascularization'. The terms were also modified according to the different databases. The last query was updated on January 1, 2013. The references of the retrieved articles were cross-searched to identify any relevant studies that were overlooked during the electronic database search.

Inclusion criteria. The inclusion criteria for the primary studies were as follows: i) the articles included definitively diagnosed UM and normal eye tissue in humans; ii) all the eye samples with melanoma were obtained by surgery and immunohistochemical analysis was used to assess the expression of VEGF in the tumor samples; iii) the UM patients had not received immune therapy, radiotherapy or chemotherapy; iv) when multiple studies were published by the same authors or institutions, the most recent or informative was selected; and v) studies lacking clinicopathological data for meta-analysis, review articles without original data and single-case reports were excluded.

Methodological assessment. Our initial selection for all candidate studies was based on the careful screening of their abstracts by two independent reviewers (Meng Yang and Xiaocong Kuang), using a standardised data collection form, including the following items: name of first author, year of publication, ethnicity, patient gender, mean or median age, cell type, LTD, tumor size, scleral invasion, VEGF assessment method, cut-off value of VEGF positivity (\%), number of readers, blinded reading (the investigator assessing the slides was blinded to the clinical information) and number of events in each category of VEGF.

We also screened the references from the relevant literature, including all the identified studies, without including additional reviews and editorials. The reference lists of the retrieved articles were also manually searched. Disagreements were resolved by consensus between the two readers. In the instance of a persistent disagreement, the final decision was made by a third expert investigator (Jianmin Li).

We did not set a predefined minimum number of patients or a minimum duration of median follow-up for a study to be included in our meta-analysis. We did not weigh each study by a quality score, since no such score has received general approval for use in a meta-analysis, particularly of observational studies, making the evaluation of its usefulness difficult. Our readers were not blinded to the studies; however, exclusions were always decided upon without knowledge of the global result of each study. When duplicate studies were retrieved, the study involving the highest number of patients from which data could be extracted (usually the latest) was included in our meta-analysis, in order to avoid overlapping between studies.

Statistical analysis. Three categories of stratified models were analyzed. The first stratified multivariate model was performed in order to confirm whether VEGF was highly expressed in UM patients compared with the controls. The second outcome of the meta-analysis was to assess the clinicopathological characteristics of VEGF expression, including patient gender, age, cell type, LTD, tumor size and scleral invasion.
According to the clinical characteristics, the following elements were combined: high and moderate VEGF expression; poor and no VEGF expression; mixed-cell and epithelioid-cell type tumors; tumors $>15 \mathrm{~mm}$ in LTD; tumors $<15 \mathrm{~mm}$ in LTD; small and medium tumor size; patients aged $>50$ years; and patients aged $<50$ years.

All statistical analyses were performed using RevMan statistical analysis software system, version 5.2. A two-tailed $\mathrm{P}<0.05$ for the summary effect was considered to indicate a statistically significant difference. The heterogeneity of all the included studies was assessed by a statistical value $\mathrm{I}^{2}$. When $\mathrm{I}^{2}$ was $<50 \%$, the studies with an acceptable heterogeneity were considered and the fixed-effects model with the Mantel-Haenszel method was used; otherwise, a random-effects model with the DerSimonian and Laird method was adopted. The combined odds ratio (OR) was initially estimated using forest plots graphically. For each trial, the OR was estimated by a method depending on the data provided in the publication. The simplest method involved the direct retrieval of OR and its $95 \%$ confidence interval (CI) from the original article. If not available, we assessed the total numbers of events and the numbers of patients at risk in each group to estimate the OR.

The assessment of publication bias for each of the pooled study groups was performed mainly by the Egger's linear regression test. As a supplementary approach, the Begg's rank correlation was also applied to assess the potential publication bias. $\mathrm{P}<0.05$ indicated that there was no publication bias in the study.

\section{Results}

Study selection. A total of 297 references were retrieved for initial reviewing using search strategies as previously described. A total of 195 citations were excluded from the analysis after the first screening based on the abstracts or titles. Following exclusion of the articles that were out of the scope of our meta-analysis, 39 potential studies were identified for detailed evaluation. Upon further review, a further 28 studies were eliminated due to the following reasons: 9 studies overlapped with others, 7 studies measured VEGF with methods other than immunohistochemistry and 12 studies lacked informative clinical data. Finally, 11 studies on the VEGF expression were included. The selection process for the studies included in this meta-analysis is summarized in Fig. 1. and the main characteristics of the eligible studies are summarized in Table I.

Correlation of VEGF expression between UM and controls. The combined results from all the studies demonstrated that the VEGF expression in patients with UM was significantly higher compared to that in controls in 7 studies (338 patients and 99 controls; OR=16.15, 95\%CI: 8.65-30.12, $\mathrm{P}<0.00001)$. There was no statistical heterogeneity among the studies $\left[\mathrm{I}^{2}=19 \%\right.$, degree of freedom (df)=6, P=0.28; Fig. 2A].

Correlation between VEGF expression and clinicopathological characteristics

Gender. The VEGF expression in UM according to patient gender was compared in 5 studies. VEGF expression was observed in 67 of the 102 male patients $(66 \%)$ and in 62 of the 90 female patients $(69 \%)$. There was no statistical 


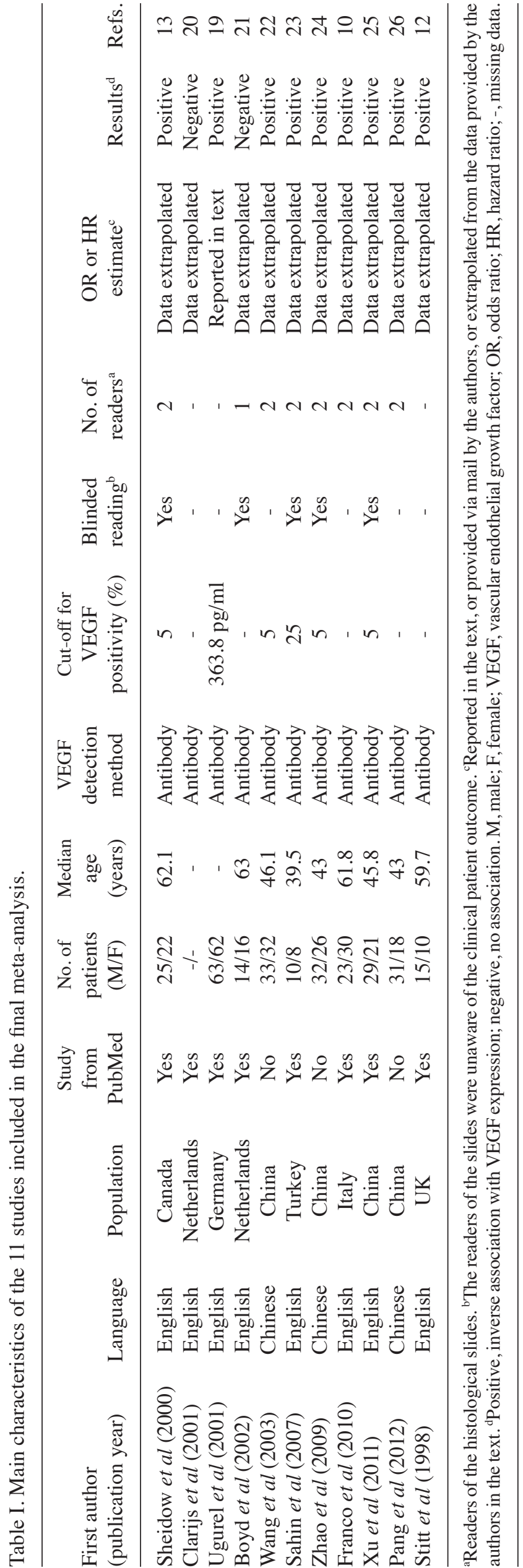

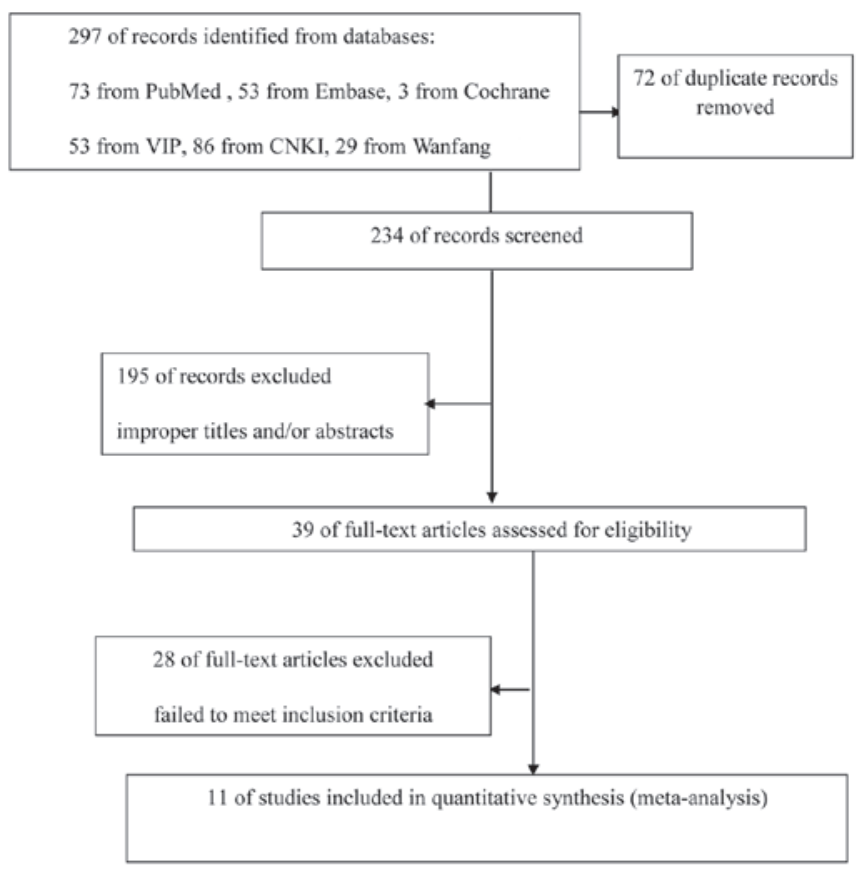

Flow chart of the literature search and inclusion strategy.

Figure 1. Selection process for the studies included in the meta-analysis.

heterogeneity among the studies $\left(\mathrm{I}^{2}=0 \%, \mathrm{df}=4, \mathrm{P}=0.66\right)$. Thus, the fixed-effects model was used for statistical analysis. No association was observed between patient gender and VEGF expression (OR=0.69, 95\% CI: 0.35-1.35, $\mathrm{P}=0.27)$.

Age. VEGF expression in UM was investigated according to patient age. The patients were divided into two groups aged $>50$ and $<50$ years. VEGF expression was observed in 116 of the $150(77 \%)$ patients in the younger group and 100 of the $165(61 \%)$ patients in the older group. No statistical heterogeneity was detected among the studies $\left(\mathrm{I}^{2}=13 \%, \mathrm{df}=6, \mathrm{P}=0.33\right)$. Thus, the fixed-effects model was used. VEGF expression was significantly higher among patients aged $<50$ years $(\mathrm{OR}=2.08$, 95\% CI: 1.19-3.62, $\mathrm{P}=0.01$ ).

Cell type. VEGF expression in UM according to cell type was compared in 7 studies. VEGF expression was observed in 99 of the 154 (64\%) mixed-and epithelioid-cell type and in 164 of the $269(61 \%)$ spindle-cell type tumors. The VEGF expression was lower in the spindle-cell type tumors compared to the other types (OR=0.54, 95\% CI: 0.32-0.90, $\mathrm{P}=0.02$ ). No statistical heterogeneity was detected among the studies $\left(\mathrm{I}^{2}=0 \%\right.$, df $=6, \mathrm{P}=0.94$; Fig. $\left.2 \mathrm{~B}\right)$.

LTD. The VEGF expression in UM according to LTD was compared in 5 studies. VEGF expression was observed in 122 of the $182(67 \%)$ tumors with an LTD of $>15 \mathrm{~mm}$ and in 69 of the $86(80 \%)$ tumors with an LTD of $<15 \mathrm{~mm}$. There was statistical heterogeneity among the studies $\left(\mathrm{I}^{2}=51 \%, \mathrm{df}=4\right.$, $\mathrm{P}=0.09$ ). Thus, the random-effects model was used for statistical analysis. No association was observed between LTD and VEGF expression (OR=0.43, 95\% CI: 0.15-1.24, $\mathrm{P}=0.12$ ).

Tumor size. VEGF expression in UM according to tumor size was compared in 4 studies. VEGF expression was observed in 47 of the $72(65 \%)$ small- and medium-sized and in 121 of the $150(81 \%)$ large-sized tumors. VEGF expression was significantly higher in large-sized tumors $(\mathrm{OR}=0.30$, 


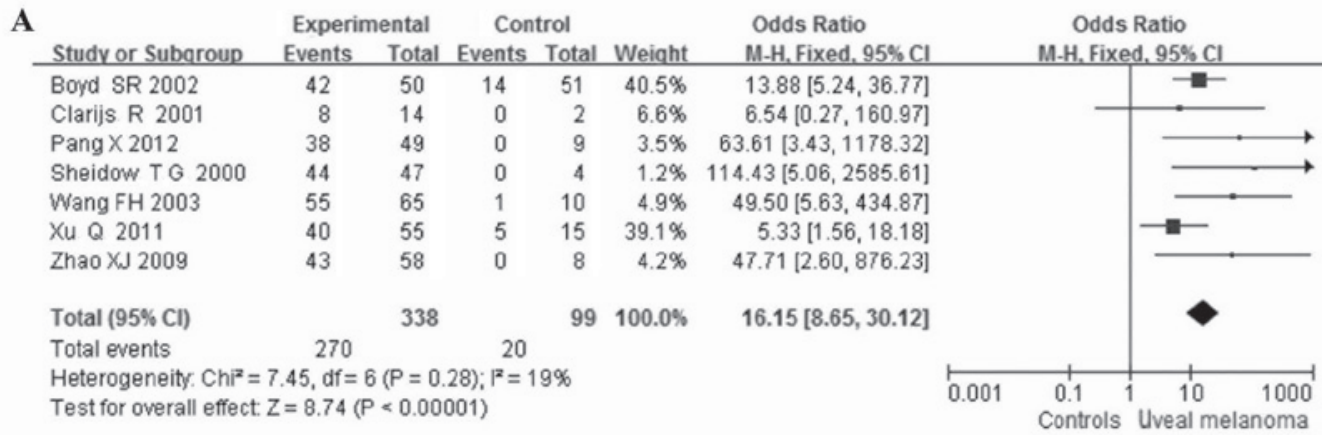

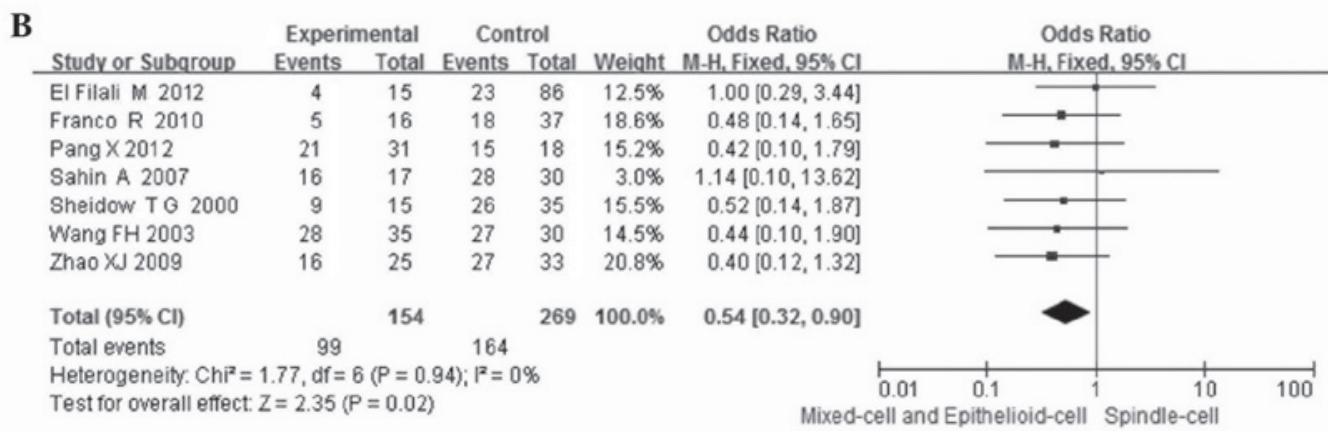

Figure 2. Meta-analysis of (A) the association of vascular endothelial growth factor (VEGF) expression with uveal melanoma and comparison with controls and (B) the association between VEGF expression and tumor cell type.

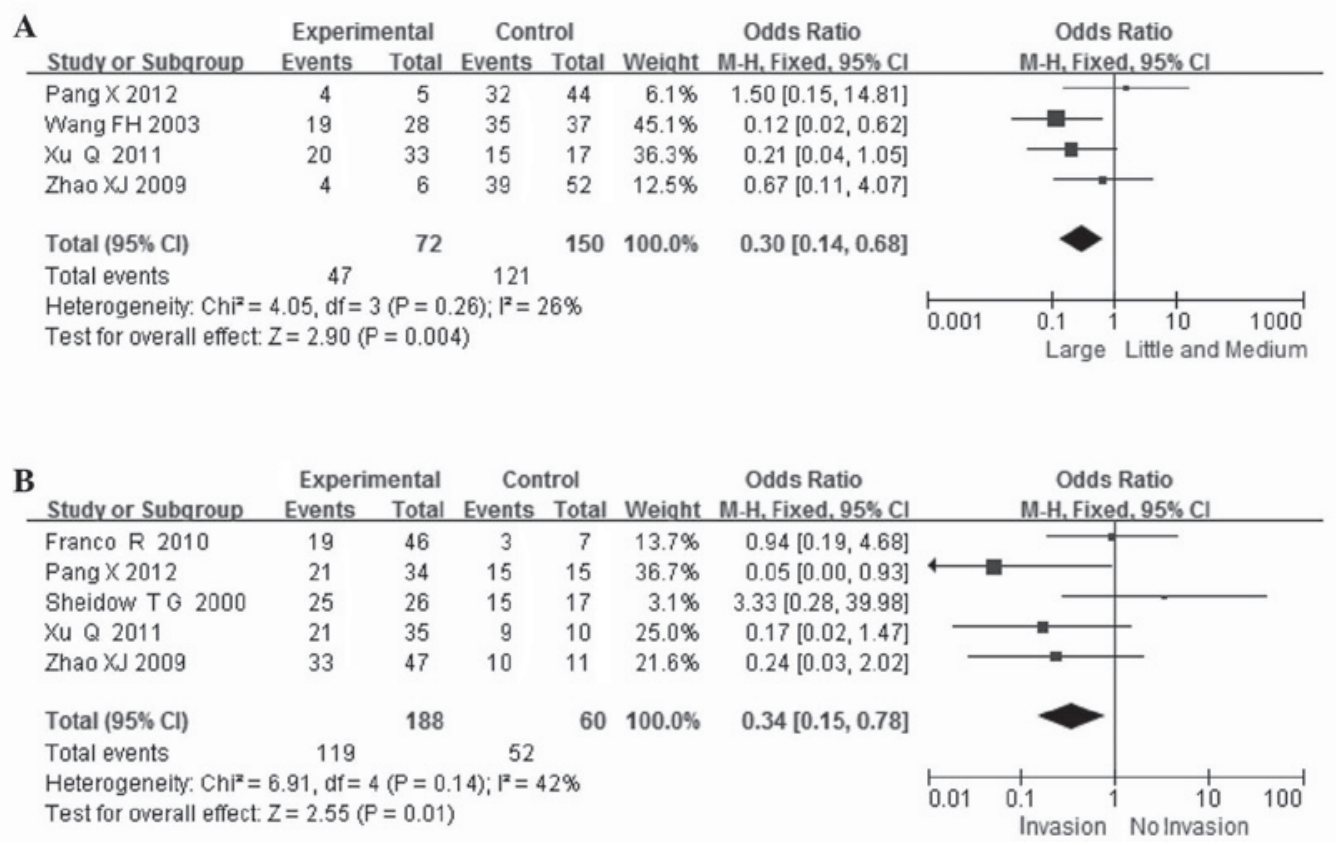

Figure 3. Meta-analysis of (A) the association between vascular endothelial growth factor (VEGF) expression and tumor size and (B) the association between VEGF expression and scleral invasion.

95\% CI: 0.14-0.68, $\mathrm{P}=0.004)$. There was no statistical heterogeneity among the studies $\left(\mathrm{I}^{2}=26 \%, \mathrm{df}=3, \mathrm{P}=0.004\right.$; Fig. 3A).

Scleral invasion. VEGF expression in UM according to scleral invasion was compared in 5 studies. VEGF expression was observed in 119 of the $188(63 \%)$ patients without and in 52 of the $60(87 \%)$ patients with scleral invasion. VEGF expression was significantly higher in patients who exhibited scleral invasion $(\mathrm{OR}=0.34,95 \% \mathrm{CI}$ : $0.15-0.78, \mathrm{P}=0.01)$. There was no obvious statistical heterogeneity among the studies $\left(\mathrm{I}^{2}=42 \%\right.$, $\mathrm{df}=4, \mathrm{P}=0.14$; Fig. 3B).

Sensitivity analysis and publication bias. The influence analysis revealed that no individual study significantly affected the pooled ORs and CIs. When each study was sequentially 
removed and the meta-analysis was repeated with the remaining studies, the pooled OR remained essentially the same. However, the studies including analyses of VEGF expression according to patient gender, age, cell type, LTD, tumor size and scleral invasion, were <9. The Egger's linear regression and Begg's tests were not used to investigate publication bias.

\section{Discussion}

Since the VEGF was identified, there has been an increasing number of studies on the association between VEGF and cancer research. It was demonstrated that VEGF expression is strongly positive in a variety of human malignant tumors in the mRNA and/or the protein level (11). Pigment and blood vessels are abundant in uveal tissue. Tumor cells are mainly transferred through the blood; therefore, angiogenesis is crucial for the development of the tumor. However, currently available studies on VEGF expression in UM are sparse and the results obtained are inconsistent. Kvanta et al (5) reported that VEGF mRNA and protein expression were strongly positive in retinoblastoma, although there was no VEGF expression in UM. Stitt et al (12) investigated the expression of VEGF mRNA, VEGF protein and its receptor in retinoblastoma or $\mathrm{UM}$ and reported a strongly positive VEGF expression in the tumor; the VEGF expression rate was higher compared to that in the uninvolved normal retina, choroid and iris. Sheidow et al (13) reported that 37\% (16/43) of the patients with choroidal melanoma developed distant metastases within the 10-year follow-up, but did not confirm the association of VEGF expression with distant metastasis, although the expression of VEGF in the choroidal melanoma cells was strongly positive (94\% positivity rate).

To the best of our knowledge, this is the first comprehensive and detailed meta-analysis assessing the association between the expression of VEGF in UM and clinicopathological characteristics. The stratification of the baseline patient characteristics, including gender, age, cell type, LTD, tumor size and scleral invasion, our results demonstrated that VEGF expression is of clinicopathological value in UM, which may increase the predictive accuracy of prognosis in UM patients.

Our results demonstrated that high VEGF expression, as detected by immunohistochemistry, was confirmed in patients with UM according to evidence-based medicine. Notably, in the subgroup meta-analysis, we also observed that VEGF overexpression was correlated with a patient age of $<50$ years $(\mathrm{P}=0.008)$, which may explain its prognostic effect to some extent. Similar findings were also reported by other studies on age (14). However, further studies are required to assess the association of VEGF expression with age. We also observed that VEGF expression was significantly higher in mixed-cell and epithelioid-cell tumors and lower in spindle-cell tumors.

The association between tumor size and VEGF expression was not clearly determined. Our meta-analysis indicated that the VEGF expression was increased in large-sized tumors and decreased in small- and medium-sized tumors. We also demonstrated that VEGF expression was significantly higher in patients with scleral invasion. A collaborative ocular melanoma study in the USA investigated 1,091 enucleation specimens and reported a scleral invasion rate of 55\% (15).
The data mentioned previously questions the feasibility and safety of the implementation of local tumor resection and may explain the high recurrence rate following tumor resection in patients with UM (16). Those findings may also explain the increased formation of tumor microvasculature with the increasing degree and local infiltration of UM.

High VEGF expression induces the formation of a rich vascular network and nutritious environment, which is an active process that requires degradation of the extracellular matrix and increase in vascular permeability of blood and lymphatic vessels, favoring the progression of tumor cells into the blood and lymphatic vascular space (17). This may offer an explanation for the observed strong statistical association of VEGF overexpression with tumor invasion and metastasis (18).

The present study had several limitations. Firstly, although we did not detect significant publication bias among the studies, apart for erythrocyte sedimentation rate (ESR), it is uncertain whether the cases are comparably representative. Furthermore, the studies were observational and, therefore, more prone to biases compared with prospective randomized controlled studies. We detected publication bias for $\mathrm{ESR}(\mathrm{P}=0.2)$ and there may be missing information which may reflect a negative or a more conservative association of ESR with DR4. More samples are required to validate the reliability of our conclusions.

In conclusion, our meta-analysis demonstrated that VEGF may be a marker that may enable earlier identification of high-risk patients and guide clinical decision-making regarding therapy and outcome. The evaluation of serum levels of VEGF expression may therefore play an important role in selecting melanoma patients for antiangiogenic therapy (19).

However, our conclusion should be interpreted with caution, since this analysis would be ideally performed on series of patient data. Further investigation into this subset of patients from other studies should assess the generalization of results prior to the implementation of VEGF in the routine clinical management of UM patients. Future prospective studies investigating the association of VEGF expression with survival or response to antiangiogenic therapy are required. The assessment of these angiogenic markers may be better standardised in future studies.

\section{Acknowledgements}

This study was supported by the Funds for Guangxi Zhuang Autonomous Region Science and Technology Hall (grant no. 1140003B-86)

\section{References}

1. Dobner BC, Riechardt AI, Joussen AM, Englert S and Bechrakis NE: Expression of haematogenous and lymphogenous chemokine receptors and their ligands on uveal melanoma in association with liver metastasis. Acta Ophthalmol 90: e638-e644, 2012.

2. Petousis V and Finger PT: Current methods for the diagnosis and treatment of choroidal melanoma. US Ophthalmic Rev 5: 62-69, 2012.

3. Woodman SE: Metastatic uveal melanoma: biology and emerging treatments. Cancer J 18: 148-152, 2012.

4. Barak V, Pe'er J, Kalickman I and Frenkel S: VEGF as a biomarker for metastatic uveal melanoma in humans. Curr Eye Res 36: 386-390, 2011

5. Kvanta A, Steen B and Seregard S: Expression of vascular endothelial growth factor (VEGF) in retinoblastoma but not in posterior uveal melanoma. Exp Eye Res 63: 511-518, 1996. 
6. Musumeci F, Radi M, Brullo C and Schenone S: Vascular endothelial growth factor (VEGF) receptors: drugs and new inhibitors. J Med Chem 55: 10797-10822, 2012.

7. Erdei E and Torres SM: A new understanding in the epidemiology of melanoma. Expert Rev Anticancer Ther 10: 1811-1823, 2010.

8. El Filali M, van der Velden PA, Luyten GP and Jager MJ: Anti-angiogenic therapy in uveal melanoma. Dev Opthalmol 49: $117-136,2012$

9. Spagnolo F, Caltabiano G and Queirolo P: Uveal melanoma. Cancer Treat Rev 38: 549-553, 2012.

10. Franco R, Botti G, Mascolo M, et al: CXCR4-CXCL12 and VEGF correlate to uveal melanoma progression. Front Biosci (Elite Ed) 2: 13-21, 2010

11. Berse B, Brown LF, Van De Water L, Dvorak HF and Senger DR Vascular permeability factor (vascular endothelial growth factor) gene is expressed differentially in normal tissues, macrophages, and tumors. Mol Biol Cell 3: 211-220, 1992.

12. Stitt AW, Simpson DA, Boocock C, Gardiner TA, Murphy GM and Archer DB: Expression of vascular endothelial growth factor (VEGF) and its receptors is regulated in eyes with intra-ocular tumours. J Pathol 186: 306-312, 1998.

13. Sheidow TG, Hooper PL, Crukley C, Young J and Heathcote JG: Expression of vascular endothelial growth factor in uveal melanoma and its correlation with metastasis. Br J Ophthalmol 84 750-756, 2000

14. Perrone G, Santini D, Vincenzi B, Zagami M, La Cesa A, Bianchi A, Altomare V, Primavera A, Battista C, Vetrani A, Tonini $\mathrm{G}$ and Rabitti C: COX-2 expression in DCIS: correlation with VEGF, HER-2/neu, prognostic molecular markers and clinicopathological features. Histopathology 46: 561-568, 2005.

15. Schori H, Kipnis J, Yoles E, WoldeMussie E, Ruiz G, Wheeler LA and Schwartz M: Vaccination for protection of retinal ganglion cells against death from glutamate cytotoxicity and ocular hypertension: implications for glaucoma. Proc Natl Acad Sci USA 98: 3398-3403, 2001

16. Damato BE, Paul J and Foulds WS: Predictive factors of visual outcome after local resection of choroidal melanoma. Br J Ophthalmol 77: 616-623, 1993.
17. Schwarz MA and Cleaver OB: Development of the pulmonary endothelium in development of the pulmonary circulation: vasculogenesis and angiogenesis. In: The Pulmonary Endothelium: Function in Health and Disease. Voelkel NF and Rounds S (eds). 1st edition. Wiley-Blackwell, Chichester, pp3-24, 2009.

18. Ding GX, Feng CC, Song NH, et al: Paraneoplastic symptoms: Cachexia, polycythemia, and hypercalcemia are, respectively, related to vascular endothelial growth factor (VEGF) expression in renal clear cell carcinoma. Urol Oncol: Apr 23, 2012 (Epub ahead of print).

19. Ugurel S, Rappl G, Tilgen W and Reinhold U: Increased serum concentration of angiogenic factors in malignant melanoma patients correlates with tumor progression and survival. J Clin Oncol 19: 577-583, 2001.

20. Clarijs R, Schalkwijk L, Ruiter DJ and de Waal RM: Lack of lymphangiogenesis despite coexpression of VEGF-C and its receptor Flt-4 in uveal melanoma. Invest Ophthalmol Vis Sci 42: 1422-1428, 2001

21. Boyd SR, Tan DS, de Souza L, et al: Uveal melanomas express vascular endothelial growth factor and basic fibroblast growth factor and support endothelial cell growth. Br J Ophthalmol 86: 440-447, 2002.

22. Wang FH, Li B, Sun XL, et al: Correlation of tumor angiogenesis with clinicopathologic prognostic parameters in choroidal melanoma. Chin J Ophthalmol 39: 68-72, 2003 (In Chinese).

23. Sahin A, Kiratli H, Tezel GG, Soylemezoglu F and Bilgic S: Expression of vascular endothelial growth factor A, matrix metalloproteinase 9 and extravascular matrix patterns in iris and ciliary body melanomas. Ophthalmic Res 39: 40-44, 2007.

24. Zhao XJ, Li B, Li LQ, Gao F and Sun XL: Relationship of survivin and VEGF with angiogenesis in choroidal melanoma. Chin Ophthal Res 27: 770-773, 2009 (In Chinese).

25. Xu Q, Zhao GQ, Zhao J, et al: Expression and significance of factors related to angiogenesis in choroidal melanoma. Int J Ophthalmol 4: 49-54, 2011.

26. Pang X, Yunhui Q and Yuewe M: Expressions of A disintegrin and metalloproteinase 9 and vascular endothelial growth factor in ocular malignant melanomas. Rec Adv Ophthalmol 32: 620-623, 2012 (In Chinese). 